\title{
PHYSICIAN ASSISTANT STUDENTS AT TRAKIA UNIVERSITY, MEDICAL FACULTY OF STARA ZAGORA ABOUT THEIR CHOICE OF SPECIALTY
}

\author{
P. Vracheva* \\ Department of "Health Care”, Medical Faculty, Trakia University, Stara Zagora, Bulgaria
}

\begin{abstract}
The aim of the study is to investigate the choice of specialty by the physician assistant students at the Medical Faculty of Stara Zagora, as followers of the feldschers in Bulgaria, whose training was suspended in 1999, with subsequent restart of the profession under the name "Physician assistant" in 2014. Methods: During November - December 2016 an empirical sociological study was conducted, which consisted of a semi-structured face-to-face interview with all 42 students of the specialty. Thirteen students chose the specialty as first preference. "Physician assistant" as a future profession is a second preference for 10 , subsequent for 3 , and 14 have been redirected from another specialty. The majority of the respondents identify themselves as well informed in advance about the profession. More than half of the students (25) personally know feldschers, realizing an independent, "indirectly" influenced by their professional activity choice of specialty, identified as "conscious" for 30 and "occasional" for 12 of them. Conclusion: The context of the results allows the choice of "Physician assistant" specialty by the student respondents to be considered as free and conscious for the majority of them, motivated by their awareness of the profession and possibilities for realization of the staff.
\end{abstract}

Key words: students, physician assistants, specialty, awareness, professional realization, interview.

\section{INTRODUCTION}

The choice of a profession is an important step in everyone's life, determining his future realization and sense of success and satisfaction, which is usually done relatively early, in the absence of life experience and knowledge. It is quite difficult for a young person to be fully aware of the importance of their preference. Researchers who have studied the motivation behind the choice of a profession claim that on the question "How did you choose your future profession" only a few people gave a reasonable answer for their choices. Most of them have stated that they have been guided by their parents' wishes and ambitions, made a choice as a "career inheritance", others defined their choice as a spontaneous act or attracted by the financial security which the profession provides $(1,2$, 3). Probably, in today's dynamic living conditions, factors which contribute to the importance and influence of the worldview and value orientation of the young people in

\footnotetext{
*Correspondence to: Penka Vracheva, Trakia university, Medical faculty of Stara Zagora, Department of "Health care", 11 Armeyska Street, 6000 Stara Zagora, Phone: +359 889141 437, Email:p_vracheva@abv.bg
}

Bulgaria are the socio-economic transition in the country and the belonging to the European Union, the applied principles of market economy in the globalizing world with the opportunities for cross-border movement and professional realization. At the same time there are certain qualities which are required for practicing the medical profession. The difficult course of education at the higher medical schools and the so called lifelong learning, the challenges in the professional activity and specialization, the dynamics in the health needs of the society with specific expectations from the professionals are a serious premise for that the choice of medical specialty to be responsible, conscious and highly desired by the people who aim at a professional career in the health system. The physician assistant students, in the role of student applicants, who have declared their desire to learn and master a medical profession unfamiliar to Bulgaria under the name "Physician assistant", face a real challenge and responsibility for their future realization as professionals.

In recent years, the "Physician assistant" profession has been established in many countries, as part of the strategy to overcome 
staff shortage and unequal distribution of labor resources in healthcare - a problem identified by the World health organization (WHO) as a global phenomenon $(4,5,6)$. In Bulgaria, following the international experience, "Physician Assistant" students are trained at the Faculty of Public Health (FPH), Medical University of Sofia and the Medical Faculty (MF), Trakia University of Stara Zagora with a decision of the Council of Ministers (CS) from 2014 in compliance with all the normative documents for the training of medical specialists in the country $(7,8)$. The future physician assistants, as followers of the medical feldschers, whose training was suspended in 1999, will be cadres from the "Health Care Directorate" with a training based on a medical model (9). The "Physician assistant" profession in Bulgaria is perceived as a restart of the "feldscher" profession with a change of the name imposed by the need for recognition of cadres outside the country under the contemporary conditions of cross-border movement of medical professionals with the possibility of professional realization.

As researchers, we are interested in the choice of "Physician assistant" specialty by the students who are being educated after the resurrection of the newly named profession.

\section{METHODS}

The research about the choice of specialty from the physician assistant students is a part of empirical sociological study which was conducted during the period November December 2016. The semi-structured face-toface interview method has been applied in order to obtain primary sociological information. The subject of study have been the students of the "Physician assistant" specialty, Department of Health Care at the Medical Faculty, Trakia University- Stara Zagora. All current students have been invited to participate in the interview. The number of those who have responded to the invitation and participated in the survey is $42 \quad(100 \%$ responsiveness) and they have been in different years of study as it follows: first-year students -10 , second -8 and 24 students in third year.

\section{Instruments}

The questionnaire for the interview has been made by the researchers according to the preset objective and tasks. The same was approved by the Ethics Committee of Medical Faculty, Trakia University - Stara Zagora (Protocol № 4/25.04.2017).

The semi-structured face-to-face interview method has been preferred for the survey, given the small number of respondents and the 224

Trakia Journal of Sciences, Vol. 16, Suppl. 1, 2018 opportunities it provides as a qualitative method for obtaining reliable and comprehensive information, as the interviewer predisposes the respondents, creating an atmosphere of trust for sharing frank opinions on the questions asked.

The interview, which has been of 30-40 minutes duration, occurred in an education room at the Department of Health Care at the Medical Faculty, Stara Zagora, without having the students' learning process disturbed. The preliminary plan and time allocation for a meeting with the respondents have helped for a good organization and created a relaxed environment, provided enough time for interviewing and met the anonymity requirements regarding the opinions which the students shared on the questions posed. I would like to note that the interview has been conducted by a student-respondents' lecturer which could have some influence on the answers.

In this material, I present some of the results of the interview, concerning the choice of a specialty made by the physician assistant respondents. The questions that have been asked aim to enlighten the problem from different positions (questions 2, 3, 4, 5, 6 and 7 of the questionnaire).

\section{Statistical processing and analysis}

A database has been created using Microsoft Excel 2010 and the Statistica 10 (http://www.statsoft.com). There have been used methods of descriptive statistics in accordance with the specifics of the sociological survey methodology applied on a small number of respondents with the preference of absolute frequency distributions.

\section{RESULTS AND DISCUSSION}

Socio-demographic characteristics - age, sex, permanent residence of the student respondents

- Age: the share of students aged between 21 and 30 is the biggest - 22. The youngest students are 18 years old - two, and the oldest is 39 years old. The average age of the students is 22.8 years: a relatively homogeneous group of young people;

- Distribution by gender: there is a slight difference in the ratio of the students based on their gender - 22 men and 20 women;

- Permanent residence - the majority of the students (33) are from town and 9 from village.

In the study we have examined which in row has the choice of "Physician assistant" 
specialty been made, having in mind the desires which the students have declared in their applications during their student admission campaign. In answer to the question: "Your admission in this specialty is" we have established the following information:

- for 13 students the "Physician assistant" specialty is a first preference, and for 2 first and only;

"Physician assistant" is:

- a second wish for a future profession for 10 of the current students;

- a subsequent wish for 3 students;

- redirected from another specialty - 14 students, (13 from the "paramedic" specialty). In 2014, by a decision of the Council of Ministers, 10 students were admitted to the Medical Faculty, Trakia University. The training course in the specialty, according to the Higher Education Law in Bulgaria and all the requirements for education of specialists in the Health Care Directorate, is four years with the acquisition of Bachelor's Degree and professional qualification "Physician assistant". During 2013/14 academic year there was an admission of students in "Paramedic" specialty at the Medical College of Stara Zagora, whose education was suspended in 2014 by a decision of the Ministry of Health. The students were offered an opportunity to continue their education by choosing another specialty: "physician assistant", "nurse" or "midwife" with Bachelor's degree from "Department of Health Care" at the Medical Faculty, Trakia University or "rehabilitation therapist" at the Medical College of Trakia University with the acquisition of a "Professional Bachelor's Degree".

With the study we have found out that 13 physician assistant students have made a choice and have entered the specialty "Physician assistant" with a Bachelor's degree after the discontinued education of the "Paramedic" specialty - a Professional Bachelor's degree. They began their training in the "Physician assistant" specialty, as redirected from another specialty, along with the newly admitted physician assistants - 10 students. The opportunity for the former paramedic students to continue their education as physician assistants is interpreted not only as a chance provided by the decision of the Ministry of Health but also as a choice of students who actually have the real opportunity to choose between several specialties, respectively future professions. With the interview we have understood that three of the students admitted in the "Paramedic" specialty had an initial desire for the "Physician assistant" specialty, but their examination marks proved to be insufficient to meet their goal, which they subsequently accomplished through their second opportunity "Redirected from paramedic".

One student has suspended his current education of his own will and has chosen to redirect to the "Physician assistant" specialty.

Analyzing the results of the study, I interpret the choice of the "Physician assistant" specialty by the students as corresponding in its most parts to the desire of the students to be future professionals - physician assistants. The three students enrolled in the specialty after a classification on "following desire" are considered as admitted in the specialty according to the personal opportunities which their examination marks offered.

We have investigated the extent to which the choice of the "Physician assistant" specialty is motivated, based on the knowledge of the profession, which is new and unfamiliar to Bulgaria under the name "Physician assistant". On the question: "Did you have any prior information about the "Physician assistant" profession" we have established the following results:

- half of the students in the specialty (21) responded with "yes" - they had preliminary information about the profession.

- 15 students defined themselves as "partially" informed

- only 6 of the respondents answered the question with "no", which means they had no prior information about the profession.

Given the opportunity to share a free answer, the students in the interview have talked about the sources of information that are diverse: literature for the "Physician assistant" profession in other countries, relatives and friends who work in the medical sphere abroad, people who are acquainted with the professional competence of the physician assistants, university lecturers, students from other specialties, information about the specialty from internet sites. It should be noted that the assessment of the prior knowledge about the profession regarding the profession, in response to the question "Did you have preliminary information about the "Physician assistant" profession", is subjective, based on the students' personal understanding of what does it mean to be informed. We have been persuaded in this during the course of the interview where some of the students who identified themselves as "partially" informed turned out to be no less familiar with the "Physician assistant" profession than the 
students who have chosen the "informed" answer.

The physician assistants in Bulgaria are followers of the professionals qualified as "feldscher". On the question: "Do you have a relative or an acquaintance working as feldscher or physician assistant (working abroad)?" more than half of respondents (25) have answered with "yes", with "no" - 17 students respectively.

Only students (25) who know professionals qualified as feldschers and physician assistants have answered the question: "Have your choice of specialty been influenced by their opinion?". The results are:

Eight of the students have answered with "yes" to the question if they have been influenced in their choice of specialty, two of whom have defined their choice of profession as having been made under the direct influence of feldscher relatives. The other six have determined their choice as "indirectly" influenced. That is to say, knowing feldschers and being familiar with their professional activity, they have decided to choose the profession for their own. For example, a student whose grandmother is a feldscher, has observed her work since childhood. She has witnessed patients' gratitude to her grandmother's professionalism many times, and gradually over the years has formed the desire to choose her grandmother's profession for her own.

The results of the study give reason to consider the choice of the "Physician assistant" specialty by the student respondents as free and somehow conscious for the majority of them.

As researchers we are interested in the students' satisfaction towards the choice of specialty, defining their future profession. On the question: "Are you satisfied with the choice of specialty at this stage of your education?" according to the answers of the satisfaction assessment - "yes", "no", "I still cannot decide" we obtain these results:

- the majority of the physician assistant students (32) have responded with "yes",

- 10 students have answered "I still cannot decide" -6 of them have been in their first university year, one - in the second and 3 students have been in their third year.

- The answer "no", about the satisfaction of choosing a specialty, has not been present in the survey results.
The students, who responded with "yes" and "no" to the question about the satisfaction of choosing a specialty, were offered with an opportunity to share their opinion in a free answer. According to the results, 32 students had this opportunity - those who were satisfied with their choice of specialty. I present some of the arguments in the form of quotes: "The education meets my interests and expectations"; “... The training is of a high level and will allow me to prepare myself as a specialist."; “... The specialty is good, giving an opportunity for realization in various structures of the health system. The training is at a high level with considerable requirements, which will contribute to our formation as specialists ..."; "The training in the specialty corresponds to the information about the specialists which I had in advance...”.

I assume that first-year students need more time to assess their satisfaction with the choice of specialty.

In the study we plan to investigate how many of the students, who were dissatisfied and still hesitant about their assessment of the satisfaction with the choice of specialty, are willing to apply for medicine or another specialty in a subsequent student admission campaign. The absence of a negative answer raised the question to 10 students who have indicated that they were "still hesitate" about the satisfaction of choosing a specialty. The question "Would you apply for medicine or another specialty in a subsequent student admission campaign" was not raised to the 32 students who were satisfied with their choice at that stage of the education and were supposed to prefer omitting the challenge of applying again. The responses of the 10 students who were "still hesitate" about the satisfaction of choosing a specialty are distributed as follows:

- "no" - I would not apply for another specialty - 6 students;

- "yes" or willingness to apply in a subsequent student admission campaign was answered by 3 students. They were willing to test their abilities in "Medicine", but in case of failure they would continue their education in the "Physician assistant" specialty.

- At the time of the study only 1 student was still hesitating about the opportunity to re-apply in a subsequent student admission campaign.

The results of the satisfaction with the choice of specialty and plans of the students for an application in another specialty give me the opportunity to assume that at the time of the 
study, the students in the "Physician assistant" specialty evaluated their choice of specialty as successful.

The choice of "Physician assistant" specialty directly corresponds to the choice of profession - a reason for examining the opinion of the physician assistant students on the question "Do you determine your choice of "Physician assistant" profession as "conscious" or "occasional"" we establish the following results:

- 30 students, of all 42 respondents, determined their choice as "conscious";

- 12 students defined it as "occasional". These were students from the former "paramedic" specialty, who pointed out the opportunity given to them after the discontinued training of the initially chosen specialty as an "occasional choice" regarding the "Physician assistant" specialty, but emphasized that subsequently this choice became realized. The benefits which the profession provides made them feel "lucky" in terms of the chance given to them by this opportunity.

In the presentation and discussion of the results about the choice of "Physician assistant" specialty made by the physician assistant students at the Medical Faculty, Trakia university of Stara Zagora, I take into consideration the peculiarities of the study such as design, methodology with its strengths and weaknesses, as well as the peculiarities of the students at a different stage of their education. At the time of the study, second and third-year students had studied the majority of the subjects in the curriculum, which allowed them to build up an opinion with a subsequent reasoned assessment of the satisfaction with the choice of specialty. After one-year training course in the two specialties, the "Physician assistant" students who were initially in the "paramedic" specialty have a real basis for comparison of the education with an assessment of the professional opportunities, which the both qualifications give them. Accordingly, first-year respondents were still at the beginning of their education, which determines the logical response to the satisfaction of specialty choice of most of them - "I still cannot decide". I discuss the results of the interview in direction of choosing the "Physician assistant" specialty in the context of the students' preference towards the specialty, respectively the profession, based on the professional activity of the physician assistants in other countries and the Bulgarian experience of the cadres with "feldscher" qualification, to which the student respondents appear to be followers. Bulgaria's membership in the European Union with the subsequent opportunities for cross-border movement and successful realization for the future professionals in the conditions of globalization of the "Physician assistant" profession plays a role in the specialty preference of the students, as a group of young people.

\section{CONCLUSION}

The results of the empirical sociological study with the physician assistants allow me to consider the choice of "Physician assistant" specialty by the student respondents as free, successful at the time of the study and "conscious" to a certain degree for the majority of them.

The physician assistant students at the Medical Faculty, Trakia university of Stara Zagora are aware of the profession and the opportunities for realization of the personnel with this qualification. More than half of the student respondents are familiar with the feldschers' professional activity in Bulgaria because of relatives and acquaintances in this professional sphere and realize the situation of being their followers with the name "Physician assistant", after the discontinued training of feldschers. The independent choice of specialty for the majority of students, based on the awareness of the "Physician assistant" profession, presupposes the investigated satisfaction with the choice of specialty of a large number of respondents, with a refusal of applying in another specialty in a subsequent university admission campaign. This logically corresponds with the high result for the "conscious" choice of "Physician assistant" profession by the students.

\section{ACKNOWLEDGEMENTS}

I would like to express my gratitude towards the students, who participated in the interview, for their responsiveness and readiness to share personal opinions on the issues that have been examined.

\section{NOTES}

The interview's questionnaire and the Protocol of the Ethics Committee of the Medical Faculty, Trakia University Stara Zagora are available at the author of the manuscript.

\section{REFERENCES}

1. Ilieva, S., Organizational Development, Sofia, p. 265, 2006

2. Kazakov, A., Human Capital, Formation, Functioning and Investment Solutions, UNWE, Sofia, p. 320, 2010 
3. Monograph, Karabaylova, S., Condition and development of the human potential, Sofia, Classics and Style, p. 290, 2004

4. National Statistical Institute, Healthcare Specialists in "Health care" institutions by statistical regions and areas, available at: http://www.nsi.bg

5. AAPA, Global Application of Physician Assistants, available on: http://www.aapa.org.

6. Vracheva P., V. Vrachev, International experience in the development of profession „Physician assistant", General medicine, 19, 2, 63-68, 2017
7. Regulation № 21 on implementation of the Credits accumulation and transfer system in higher education institutions (SG Issue 89/12.10.2004);

8. Vracheva P., SWOT analysis of the students' education in „Physician assistant“ specialty at Trakia University - Stara Zagora, Health care, 1: 33-38, 2017

9. Qualification characteristics of the "Physician assistant" specialty, Bachelor's degree, Department of Health Care, Medical Faculty, Trakia University, approved at Academic Council Meeting of Trakia University, Protocol № 8 / 26.10.2016. 